\title{
Virulence-Associated Genes and Genetic Diversity of Avian Pathogenic (APEC) and Fecal (AFEC) E. Coli Isolates From Chickens
}

\section{Zhen Zhu}

Hebei University of Engineering

Mingze Cao

Hebei University of Engineering

Weiwei Wang

Lanzhou Institute of Husbandry and Pharmaceutical Sciences of CAAS

\section{Qiqi Zhu}

Hebei University of Engineering

\section{Chengye Wang}

Hebei University of Engineering

\section{Yongying Zhang}

Hebei University of Engineering

\section{Yuxiang Shi}

Hebei University of Engineering

Xuzheng Zhou

Lanzhou Institute of Husbandry and Pharmaceutical Sciences of CAAS

\section{Guangbin Cui}

Hebei University of Engineering

Jiyu Zhang ( $\square$ jiyuzhang123@163.com)

Lanzhou Institute of Husbandry and Pharmaceutical Sciences of CAAS

\section{Research Article}

Keywords: APEC, serotypes, VAGs, phylogroup, MLST

Posted Date: August 4th, 2021

DOI: https://doi.org/10.21203/rs.3.rs-644399/v2

License: (c) (i) This work is licensed under a Creative Commons Attribution 4.0 International License. Read Full License 


\section{Abstract \\ Background}

Avian pathogenic E. coli (APEC) is the etiologic agent of serious colibacillosis and causes extensive economic losses. To examine the genetic background of APEC, we characterized the serotypes, virulence genes, phylogenetic classification and MLST of 392 APEC and 586 AFEC strains isolated from infected chickens.

\section{Results}

The results showed that the most predominant serotypes were 078 (13.47\%), 02 (9.16\%), 018 (5.39\%), 020 (4.42\%) and O25 (4.09\%). The major serotypes 078 (13.47\%) and 02 (9.16\%) were significantly higher in the APEC isolates than in the AFEC isolates. Among the 16 analyzed virulence-associated genes (VAGs), iroN (100\%), ompT (100\%), fimC (92.46\%), iss (77.91\%) and irp2 (71.98\%) were the most frequently identified. Over half (54.85\%) of the strains possessed $>8-13$ VAGs, and $85.23 \%$ of the strains carried iroN-ompT-fimC-iss/irp2 VAG patterns. According to the phylogenetic analysis, phylogroups A (32.11\%) and B2 (31.36\%) proved to be the most prevalent phylogenetic groups in the AFEC and APEC isolates, respectively. The strains that belonged to phylogroup B2 were associated with more VAGs. Based on MLST, 46 STs belonging to 15 different clonal complexes were identified, and 4 were novel. ST88 (10.67\%) was found to be the most dominant ST, and it possessed at least 9 VAGs and belonged to phylogroups B2 or D. Furthermore, the isolates belonging to B2-078/02-ST88 were the most likely APEC isolates to be associated with epidemics, and they carried more VAGs than the other strains.

\section{Conclusions}

Our findings have enriched our knowledge of the molecular characteristics of APEC isolates from chickens, which will be important for the prevention and control of avian colibacillosis.

\section{Background}

Avian pathogenic Escherichia coli (APEC) has been reported as an etiologic agent of colibacillosis in poultry worldwide [1-3]. APEC isolates can cause severe respiratory and systemic infections that lead to a significant economic burden in the poultry industry due to increased mortality, medication costs, and condemnation of carcasses in the prevention and control of the disease [4]. Avian colibacillosis is characterized by yolk sac infection, swollen-head syndrome, septicemia, and inflammation, such as pericarditis, perihepatitis, airsacculitis, salpingitis, arthritis, and peritonitis, of different organs or a combination of these syndromes [5].

APEC infects all ages of commercial poultry and is considered a major or minor pathogen that accounts for $3-4 \%$ of the mortality of birds on a farm; however, the mechanisms underlying infection and systemic displacement by APEC are ill defined. Elucidation of the underlying molecular mechanisms of APEC pathogenicity is essential for controlling avian colibacillosis.

Recently, multiple virulence-associated genes (VAGs) have been identified that contribute to APEC pathogenesis [6]. These VAGs are involved in different steps of infection and/or adaptation, and their functions can be classified as adhesins, invasins, toxins, iron acquisition systems, and protectins/serum resistance [7-8], thereby protecting the pathogen from the host immune response and enabling its extraintestinal existence [7]. The nature and the combination of the VAGs of APEC could determine the degree of virulence and their potential to cause specific diseases in specific 
hosts. However, the importance and interaction of specific VAGs that determine the pathogenesis of APEC infections are still poorly understood [9]. Furthermore, some avian fecal E. coli (AFEC) isolated from healthy poultry may also carry certain VAGs reflecting their virulence potential.

Previous observational studies have shown that a large number of different serotypes of $\mathrm{E}$. coli, such as $01,02,05,06$, $07,09,018,024,025,036,054,078,0100$, and 0115 , are known to be detected in poultry [10-11]. Although several APEC serotypes are responsible for avian colibacillosis, the most commonly encountered APECs in different countries and on different farms are 01, 02, and 078 [12].

Other techniques, including phylogroup and multilocus sequence typing (MLST), are established tools to type APEC. E. coli isolates may be classified into four main phylogroups: $A, B 1, B 2$, and D. Among them, strains from the B2 and D phylogroups are most frequently found in APEC, while commensal intestinal strains commonly belong to the A and B1 groups [13]. MLST is a top-level genetic tool for differentiating E. coli that allows the assignment of closely related strains in clonal groups or complexes as a sequence type (ST) [14]. These standardized classifications have facilitated the identification and monitoring of pandemic strains that cause nosocomial and community outbreaks [15-16].

Several studies have described APEC strains in the literature; however, little information is available on the characteristics of avian E. coli, especially their serotype level, VAGs and molecular characterization. Therefore, the objective of this study was to determine the genetic background of highly pathogenic E. coli isolates of avian colibacillosis outbreaks and compare this information with that of AFEC strains obtained from healthy poultry.

\section{Results}

\section{Isolate collections}

In this study, a total of 2947 E. coli strains were isolated, of which 426 strains were collected from tissue and 2531 strains were collected from feces. After PCR detection, 928 E. coli strains were identified as ExPEC. The 928 ExPEC strains were further used to study the VAGs and genetic diversity among APEC and AFEC isolates. Among them, 392 APEC isolates were collected from tissues of freshly dead chickens with suspected colibacillosis, and 536 AFEC isolates with VAGs were collected from feces.

\section{Serotyping of the isolates}

All confirmed E. coli isolates were serotyped, and 28 different serotypes were identified among the E. coli strains using multiplex PCR (Table 1). Nevertheless, $6.12 \%$ and $9.70 \%$ of the E. coli isolates were not typeable as APEC and AFEC serotypes, respectively. All 28 serotypes were found in the AFEC strains, while 021, 036 and 091 were absent in the APEC strains. 
Table 1

Serogroup distribution of the APEC and AFEC strains.

\begin{tabular}{|c|c|c|c|c|c|c|c|}
\hline \multirow[t]{2}{*}{ Serogroup } & \multicolumn{2}{|c|}{ Total $n=928$} & \multicolumn{2}{|c|}{ APEC $n=392$} & \multicolumn{2}{|c|}{ AFEC $n=536$} & \multirow[t]{2}{*}{ p-value } \\
\hline & Number & Percentage & Number & Percentage & Number & Percentage & \\
\hline 01 & 26 & $2.80 \%$ & 18 & $4.59 \%$ & 8 & $1.49 \%$ & 0.005 \\
\hline 02 & 85 & $9.16 \%$ & 72 & $18.37 \%$ & 13 & $2.43 \%$ & $<0.001$ \\
\hline 04 & 30 & $3.23 \%$ & 1 & $0.26 \%$ & 29 & $5.41 \%$ & $<0.001$ \\
\hline 05 & 29 & $3.13 \%$ & 4 & $1.02 \%$ & 25 & $4.66 \%$ & 0.002 \\
\hline 06 & 24 & $2.59 \%$ & 13 & $3.32 \%$ & 11 & $2.05 \%$ & 0.231 \\
\hline 08 & 26 & $2.80 \%$ & 21 & $5.36 \%$ & 5 & $0.93 \%$ & $<0.001$ \\
\hline 09 & 35 & $3.77 \%$ & 1 & $0.26 \%$ & 34 & $6.34 \%$ & $<0.001$ \\
\hline 011 & 22 & $2.37 \%$ & 2 & $0.51 \%$ & 20 & $3.73 \%$ & 0.001 \\
\hline 015 & 17 & $1.83 \%$ & 8 & $2.04 \%$ & 9 & $1.68 \%$ & 0.685 \\
\hline 018 & 50 & $5.39 \%$ & 45 & $11.48 \%$ & 5 & $0.93 \%$ & $<0.001$ \\
\hline 020 & 41 & $4.42 \%$ & 26 & $6.63 \%$ & 15 & $2.80 \%$ & 0.005 \\
\hline 021 & 31 & $3.34 \%$ & 0 & $0.00 \%$ & 31 & $5.78 \%$ & $<0.001$ \\
\hline 024 & 31 & $3.34 \%$ & 1 & $0.26 \%$ & 30 & $5.60 \%$ & $<0.001$ \\
\hline 025 & 38 & $4.09 \%$ & 1 & $0.26 \%$ & 37 & $6.90 \%$ & $<0.001$ \\
\hline 036 & 18 & $1.94 \%$ & 0 & $0.00 \%$ & 18 & $3.36 \%$ & $<0.001$ \\
\hline 045 & 17 & $1.83 \%$ & 4 & $1.02 \%$ & 13 & $2.43 \%$ & 0.115 \\
\hline 054 & 18 & $1.94 \%$ & 2 & $0.51 \%$ & 16 & $2.99 \%$ & 0.007 \\
\hline 065 & 31 & $3.34 \%$ & 11 & $2.81 \%$ & 20 & $3.73 \%$ & 0.53 \\
\hline 078 & 125 & $13.47 \%$ & 106 & $27.04 \%$ & 19 & $3.54 \%$ & $<0.001$ \\
\hline 086 & 24 & $2.59 \%$ & 1 & $0.26 \%$ & 23 & $4.29 \%$ & $<0.001$ \\
\hline 091 & 11 & $1.19 \%$ & 0 & $0.00 \%$ & 11 & $2.05 \%$ & 0.004 \\
\hline 0100 & 21 & $2.26 \%$ & 2 & $0.51 \%$ & 19 & $3.54 \%$ & 0.002 \\
\hline 0103 & 21 & $2.26 \%$ & 2 & $0.51 \%$ & 19 & $3.54 \%$ & 0.002 \\
\hline 0115 & 20 & $2.16 \%$ & 9 & $2.30 \%$ & 11 & $2.05 \%$ & 0.801 \\
\hline 0128 & 9 & $0.97 \%$ & 6 & $1.53 \%$ & 3 & $0.56 \%$ & 0.136 \\
\hline 0147 & 29 & $3.13 \%$ & 4 & $1.02 \%$ & 25 & $4.66 \%$ & 0.001 \\
\hline 0149 & 20 & $2.16 \%$ & 6 & $1.53 \%$ & 14 & $2.61 \%$ & 0.263 \\
\hline 0157 & 3 & $0.32 \%$ & 2 & $0.51 \%$ & 1 & $0.19 \%$ & 0.392 \\
\hline Not typeable & 76 & $8.19 \%$ & 24 & $6.12 \%$ & 52 & $9.70 \%$ & 0.041 \\
\hline
\end{tabular}


The most predominant serotypes were 078 (13.47\%), 02 (9.16\%), 018 (5.39\%), 020 (4.42\%) and 025 (4.09\%) (Fig. 1A). However, the most prevalent serotypes among the APEC and AFEC strains were significantly different. Of the serotypes tested, 078 and 02 were the most frequently observed in the APEC strains, accounting for $27.04 \%$ and $18.37 \%$, respectively. 018 (11.48\%), $\mathrm{O} 20$ (6.63\%) and 08 (5.36\%) were the next most frequent. These serotypes in the APEC stains were significantly higher than those in the AFEC stains (Fig. 1B). However, 025 (6.90\%), 09 (6.34\%), 021 (5.78\%), $\mathrm{O} 4(5.41 \%)$ and $\mathrm{O} 24(5.41 \%)$ were the top five prevalent serotypes among AFEC strains and were significantly higher in the AFEC isolates than in the APEC isolates $(P<0.0001)$ (Fig. 1C).

\section{Prevalence of virulence-associated genes}

The frequencies and combinations of 16 VAGs in $928 \mathrm{E}$. coli strains (392 APEC and 536 AFEC) were assessed by PCR. The prevalence of each gene in the APEC and AFEC isolates is shown in Table 2. The iron acquisition system gene iroN and protectin/serum resistance gene ompT were carried by all of the detected strains. More than $70 \%$ of the isolates also carried fimC (92.46\%), iss (77.91\%) and irp2 (71.98\%). Gene ibeA was not detected in any strain, and papC and neuC were detected less frequently, with $1.94 \%$ and $4.74 \%$, respectively. Compared with AFEC, all the VAGs, except iroN, omp $T$ and $i b e A$, were significantly more prevalent in the APEC strains $(\mathrm{P}<0.01)$.

Table 2

Prevalence of virulence-associated genes among the APEC and AFEC strains.

\begin{tabular}{|c|c|c|c|c|c|c|c|c|}
\hline \multicolumn{2}{|c|}{ virulence-associated genes } & \multicolumn{2}{|c|}{ Total $(n=928)$} & \multicolumn{2}{|c|}{ APEC $(n=392)$} & \multicolumn{2}{|c|}{ AFEC(n = 536) } & \multirow{2}{*}{$\begin{array}{l}\mathrm{p}- \\
\text { value }\end{array}$} \\
\hline & & Number & Percentage & Number & Percentage & Number & Percentage & \\
\hline \multirow[t]{4}{*}{ Adhesins } & fimc & 858 & $92.46 \%$ & 375 & $95.66 \%$ & 483 & $90.11 \%$ & 0.002 \\
\hline & рарс & 18 & $1.94 \%$ & 17 & $4.34 \%$ & 1 & $0.19 \%$ & $<0.001$ \\
\hline & tsh & 579 & $62.39 \%$ & 292 & $74.49 \%$ & 287 & $53.54 \%$ & $<0.001$ \\
\hline & mat & 576 & $62.07 \%$ & 316 & $80.61 \%$ & 260 & $48.51 \%$ & $<0.001$ \\
\hline Invasins & $i b e A$ & 0 & $0.00 \%$ & 0 & $0.00 \%$ & 0 & $0.00 \%$ & - \\
\hline \multirow[t]{3}{*}{ Toxins } & ast $A$ & 198 & $21.34 \%$ & 115 & $29.34 \%$ & 83 & $15.49 \%$ & $<0.001$ \\
\hline & vat & 166 & $17.89 \%$ & 111 & $28.32 \%$ & 55 & $10.26 \%$ & $<0.001$ \\
\hline & hlyF & 211 & $22.74 \%$ & 123 & $31.38 \%$ & 88 & $16.42 \%$ & $<0.001$ \\
\hline \multirow{4}{*}{$\begin{array}{l}\text { Iron acquisition } \\
\text { systems }\end{array}$} & iroN & 928 & $100.00 \%$ & 392 & $100.00 \%$ & 536 & $100.00 \%$ & - \\
\hline & fyuA & 482 & $51.94 \%$ & 285 & $72.70 \%$ & 197 & $36.75 \%$ & $<0.001$ \\
\hline & $i u c D$ & 448 & $48.28 \%$ & 251 & $64.03 \%$ & 197 & $36.75 \%$ & $<0.001$ \\
\hline & irp2 & 668 & $71.98 \%$ & 342 & $87.24 \%$ & 326 & $60.82 \%$ & $<0.001$ \\
\hline \multirow{4}{*}{$\begin{array}{l}\text { Protectins/Serum } \\
\text { resistance }\end{array}$} & iss & 723 & $77.91 \%$ & 344 & $87.76 \%$ & 379 & $70.71 \%$ & $<0.001$ \\
\hline & neuc & 44 & $4.74 \%$ & 37 & $9.44 \%$ & 7 & $1.31 \%$ & $<0.001$ \\
\hline & ompT & 928 & $100.00 \%$ & 392 & $100.00 \%$ & 536 & $100.00 \%$ & - \\
\hline & cva/cvi & 135 & $14.55 \%$ & 85 & $21.68 \%$ & 50 & $9.33 \%$ & $<0.001$ \\
\hline
\end{tabular}


Based on the different combinations of VAGs, the E. coli isolates were divided into 27 virulence gene profile types (VTs) (Table 3). Each strain possessed at least 3 different VAGs, and over half (54.85\%) of the strains possessed > 8-13 VAGs. Although all the VTs were present in the APEC and AFEC strains, there were obvious differences in the distribution. Most VTs (VT14, VT18, VT20, VT22, VT24, VT26 and VT27) that carried $\geq 9$ VAGs were significantly higher in the APEC isolates than in the AFEC isolates $(p<0.01)$. In contrast, VTs (VT1-VT4) carrying $\leq 4$ VAGs were significantly more prevalent in the AFEC isolates $(\mathrm{P}<0.05)$. However, a clear difference in VT6, VT7, VT8, VT10, VT13, VT19 and VT23 prevalence between the APEC and AFEC isolates was not found $(P>0.1)$. 
Table 3

The prevalence of virulence gene types in the APEC and AFEC strains.

\begin{tabular}{|c|c|c|c|c|c|c|c|c|}
\hline \multirow[t]{2}{*}{ VT } & \multirow{2}{*}{$\begin{array}{l}\text { Virulence genes } \\
\text { profiles }\end{array}$} & \multicolumn{2}{|c|}{ Total $(n=928)$} & \multicolumn{2}{|c|}{ APEC(n = 392) } & \multicolumn{2}{|c|}{ AFEC(n = 536) } & \multirow{2}{*}{$\begin{array}{l}\mathrm{p}- \\
\text { value }\end{array}$} \\
\hline & & Number & Percentage & Number & Percentage & Number & Percentage & \\
\hline 1 & iroN-ompT-tsh & 21 & $2.26 \%$ & 3 & $0.775 \%$ & 18 & $3.36 \%$ & 0.009 \\
\hline 2 & iroN-ompT-fimC-iss & 117 & $12.61 \%$ & 20 & $5.10 \%$ & 97 & $18.10 \%$ & $<0.001$ \\
\hline 3 & iroN-ompT-fimC-irp2 & 68 & $7.33 \%$ & 21 & $5.36 \%$ & 47 & $8.77 \%$ & 0.049 \\
\hline 4 & iroN-ompT-fimC-tsh & 84 & $9.05 \%$ & 15 & $3.83 \%$ & 69 & $12.87 \%$ & $<0.001$ \\
\hline 5 & iroN-ompT-irp2-tsh & 32 & $3.45 \%$ & 9 & $2.30 \%$ & 23 & $4.29 \%$ & 0.1 \\
\hline 6 & $\begin{array}{l}\text { iroN-ompT-fimC-iss- } \\
\text { iucD-hlyF }\end{array}$ & 21 & $2.26 \%$ & 7 & $1.79 \%$ & 14 & $2.61 \%$ & 0.403 \\
\hline 7 & $\begin{array}{l}\text { iroN-ompT-iss-mat- } \\
\text { iucD-hlyF }\end{array}$ & 17 & $1.83 \%$ & 5 & $1.28 \%$ & 12 & $2.24 \%$ & 0.28 \\
\hline 8 & $\begin{array}{l}\text { iroN-ompT-fimC-iss- } \\
\text { irp2-tsh-mat }\end{array}$ & 39 & $4.20 \%$ & 13 & $3.32 \%$ & 26 & $4.85 \%$ & 0.25 \\
\hline 9 & $\begin{array}{l}\text { iroN-ompT-fimC-iss- } \\
\text { irp2-mat-cva/cvi }\end{array}$ & 20 & $2.16 \%$ & 4 & $1.02 \%$ & 16 & $2.99 \%$ & 0.042 \\
\hline 10 & $\begin{array}{l}\text { iroN-ompT-fimC-iss- } \\
\text { irp2-tsh-mat-astA }\end{array}$ & 9 & $0.97 \%$ & 2 & $0.51 \%$ & 7 & $1.31 \%$ & 0.222 \\
\hline 11 & $\begin{array}{l}\text { iroN-ompT-fimC-iss- } \\
\text { irp2-tsh-fyuA-iucD }\end{array}$ & 9 & $0.97 \%$ & 1 & $0.26 \%$ & 8 & $1.49 \%$ & 0.057 \\
\hline 12 & $\begin{array}{l}\text { iroN-ompT-fimC-iss- } \\
\text { irp2-mat-fyuA-iucD }\end{array}$ & 22 & $2.37 \%$ & 3 & 0.775 & 19 & $3.54 \%$ & 0.006 \\
\hline 13 & $\begin{array}{l}\text { iroN-ompT-fimC-iss- } \\
\text { irp2-mat-hlyF-vat }\end{array}$ & 6 & $0.65 \%$ & 1 & $0.26 \%$ & 5 & $0.93 \%$ & 0.203 \\
\hline 14 & $\begin{array}{l}\text { iroN-ompT-fimC-iss- } \\
\text { irp2-tsh-mat-fyuA- } \\
\text { iucD }\end{array}$ & 107 & $11.53 \%$ & 65 & $16.58 \%$ & 42 & $7.84 \%$ & $<0.001$ \\
\hline 15 & $\begin{array}{l}\text { iroN-ompT-fimC-iss- } \\
\text { irp2-tsh-mat-iucD-hlyF }\end{array}$ & 12 & $1.29 \%$ & 7 & $1.79 \%$ & 5 & $0.93 \%$ & 0.256 \\
\hline 16 & $\begin{array}{l}\text { iroN-ompT-fimC-iss- } \\
\text { irp2-mat-fyuA-iucD- } \\
\text { hlyF }\end{array}$ & 11 & $1.19 \%$ & 2 & $0.51 \%$ & 9 & $1.68 \%$ & 0.104 \\
\hline 17 & $\begin{array}{l}\text { iroN-ompT-fimC-iss- } \\
\text { irp2-mat-fyuA-iucD- } \\
\text { astA }\end{array}$ & 67 & $7.22 \%$ & 37 & $9.44 \%$ & 30 & $5.60 \%$ & 0.026 \\
\hline 18 & $\begin{array}{l}\text { iroN-ompT-fimC-iss- } \\
\text { irp2-tsh-mat-fyuA- } \\
\text { iucD-hlyF }\end{array}$ & 26 & $2.80 \%$ & 19 & $4.85 \%$ & 7 & $1.31 \%$ & 0.001 \\
\hline 19 & $\begin{array}{l}\text { iroN-ompT-fimC-iss- } \\
\text { irp2-tsh-mat-fyuA- } \\
\text { hlyF-cva/cvi }\end{array}$ & 5 & $0.54 \%$ & 1 & $0.26 \%$ & 4 & $0.75 \%$ & 0.313 \\
\hline 20 & $\begin{array}{l}\text { iroN-ompT-fimC-iss- } \\
\text { irp2-tsh-mat-fyuA- } \\
\text { astA-vat }\end{array}$ & 79 & $8.51 \%$ & 52 & $13.27 \%$ & 27 & $5.04 \%$ & $<0.001$ \\
\hline
\end{tabular}




\begin{tabular}{|c|c|c|c|c|c|c|c|c|}
\hline \multirow[t]{2}{*}{ VT } & \multirow{2}{*}{$\begin{array}{l}\text { Virulence genes } \\
\text { profiles }\end{array}$} & \multicolumn{2}{|c|}{ Total $(n=928)$} & \multicolumn{2}{|c|}{$\operatorname{APEC}(n=392)$} & \multicolumn{2}{|c|}{$\operatorname{AFEC}(n=536)$} & \multirow{2}{*}{$\begin{array}{l}\mathrm{p}- \\
\text { value }\end{array}$} \\
\hline & & Number & Percentage & Number & Percentage & Number & Percentage & \\
\hline 21 & $\begin{array}{l}\text { iroN-ompT-fimC-iss- } \\
\text { irp2-tsh-mat-fyuA- } \\
\text { iucD-hlyF-vat }\end{array}$ & 28 & $3.02 \%$ & 11 & $2.81 \%$ & 17 & $3.17 \%$ & 0.748 \\
\hline 22 & $\begin{array}{l}\text { iroN-ompT-fimC-iss- } \\
\text { irp2-tsh-mat-fyuA- } \\
\text { iucD-hlyF-cva/cvi }\end{array}$ & 32 & $3.45 \%$ & 23 & $5.87 \%$ & 9 & $1.68 \%$ & 0.001 \\
\hline 23 & $\begin{array}{l}\text { iroN-ompT-fimC-iss- } \\
\text { irp2-tsh-mat-fyuA- } \\
\text { iucD-astA-cva/cvi }\end{array}$ & 25 & $2.69 \%$ & 10 & $2.55 \%$ & 15 & $2.80 \%$ & 0.828 \\
\hline 24 & $\begin{array}{l}\text { iroN-ompT-fimC-iss- } \\
\text { irp2-tsh-mat-fyuA- } \\
\text { iucD-astA -neuC }\end{array}$ & 18 & $1.94 \%$ & 14 & $3.57 \%$ & 4 & $0.75 \%$ & 0.002 \\
\hline 25 & $\begin{array}{l}\text { iroN-ompT-fimC-iss- } \\
\text { irp2-tsh-mat-fyuA- } \\
\text { iucD-hlyF-vat-cva/cvi }\end{array}$ & 9 & $0.97 \%$ & 7 & $1.79 \%$ & 2 & $0.37 \%$ & 0.03 \\
\hline 26 & $\begin{array}{l}\text { iroN-ompT-fimC-iss- } \\
\text { irp2-tsh-mat-fyuA- } \\
\text { iucD-hlyF-vat-cva/cvi- } \\
\text { neuC }\end{array}$ & 26 & $2.80 \%$ & 23 & $5.87 \%$ & 3 & $0.56 \%$ & $<0.001$ \\
\hline 27 & $\begin{array}{l}\text { iroN-ompT-fimC-iss- } \\
\text { irp2-tsh-mat-fyuA- } \\
\text { iucD-hlyF-vat-cva/cvi- } \\
\text { papC }\end{array}$ & 18 & $1.94 \%$ & 17 & 4.345 & 1 & $0.19 \%$ & $<0.001$ \\
\hline
\end{tabular}

The frequencies of VAGs in most VTs showed a regular and cumulative pattern, and most strains had similar evolutionary steps; $85.23 \%$ of the strains carried iroN-ompT-fimC-iss/irp2 VAG patterns, and $62.21 \%$ of the strains possessed iroN-ompT-fimC-iss-irp2-tsh/mat VAG patterns. Hypothetical steps of VAG acquisition were illustrated according to VAG frequencies among the VTs (Fig. 2). The VT1 strains could evolve into VT4 and VT5 strains by acquiring fimC and irp2, respectively. VT2 and VT3 could evolve into VT8 by acquiring irp2-tsh-mat and iss-tsh-mat, respectively. VT8, by acquiring fyuA-iucD, could evolve into VT14, and VT11 and VT12 could also evolve into VT14 by acquiring mat and tsh, respectively. VT14 further evolved into VT18, VT21, VT22, VT25, VT26 and VT27 by obtaining corresponding VAGs. VT8 strains that had not acquired iucD later evolved into VT10, VT19 and VT20 through the acquisition of astA, fyuA-hlyF-cva/cvi and fyuA-astA-vat, respectively.

\section{Phylogenetic Classification analysis}

The phylogenetic analysis showed that phylogroups A and B2 proved to be the most prevalent phylogenetic groups, with $32.11 \%$ and $31.36 \%$, respectively, followed by phylotypes D (18.64\%) and B1 $(17.89 \%)$. Within the APEC strains, phylogroup B2 was the largest and contained over half (59.95\%) of the strains, while for the AFEC strains, $47.95 \%$ belonged to phylotype $A$ and showed the most common groups (Table 4). 
Table 4

Phylogenetic groups of APEC and AFEC strains.

\begin{tabular}{|c|c|c|c|c|c|c|c|}
\hline \multirow[t]{2}{*}{ Phylogroup } & \multicolumn{2}{|c|}{ Total $(n=928)$} & \multicolumn{2}{|c|}{ APEC $(n=392)$} & \multicolumn{2}{|c|}{ AFEC $(n=536)$} & \multirow[t]{2}{*}{ p-value } \\
\hline & Number & Percentage & Number & Percentage & Number & Percentage & \\
\hline$A$ & 298 & $32.11 \%$ & 41 & $10.46 \%$ & 257 & $47.95 \%$ & $P<0.001$ \\
\hline B1 & 166 & $17.89 \%$ & 22 & $5.61 \%$ & 144 & $26.87 \%$ & $P<0.001$ \\
\hline B2 & 291 & $31.36 \%$ & 235 & $59.95 \%$ & 56 & $10.45 \%$ & $P<0.001$ \\
\hline D & 173 & $18.64 \%$ & 94 & $23.98 \%$ & 79 & $14.74 \%$ & $P<0.001$ \\
\hline
\end{tabular}

The strains that belonged to phylogroups B2 and D were associated with more virulence genes, presenting an average of 9.64 and 9.13 virulence genes for each strain, respectively, which was significantly higher than that for the B1 (5.68) and A (5.18) groups (Table S1).

\section{MLST-based genotype analysis}

MLST was performed to analyze the genotypic diversity of E. coli isolates based on 7 housekeeping genes. According to MLST, 928 strains were divided into 46 STs that belonged to 15 different clonal complexes, and 4 were novel and found in this study (Fig. 3, Table S2). ST88 (10.67\%) was found to be the most dominant ST, followed by ST243 (8.94\%), ST461 (7.76\%), and ST142 (7.22\%), while five STs, ST65, ST118, ST122, ST141, and ST298, were less prevalent and were represented in only one AFEC strain. ST142 was the most common ST in the AFEC strains but was absent in the APEC strains. ST461, ST88 and ST243 were the three most common ST types among the APEC strains, accounting for $11.48 \%, 10.97 \%$ and $9.95 \%$, respectively. Furthermore, all of these strains possessed at least 9 VAGs and belonged to phylogroups B2 or D.

The serotype and STs of the strains exhibited epidemic preferences (Fig. 4). ST88 was the most predominant ST among the 078 and 02 serotypes, and all the APEC strains with ST88 belonged to these two serotypes. Eleven different STs of 078 strains were found, and the top five STs were ST88 (29.60\%), ST243 (20.00\%), ST461 (15.20\%), ST131 (10.40\%), and ST85 (8.80\%), which were also the most dominant STs of the APEC strains. Eighty-five 02 strains were divided into 17 STs, and ST254 (18.82\%) and ST855 (12.94\%) were the most common STs, except ST88. ST254 was the most common ST in the APEC strains but was missing in the AFEC strains among the 02 serotypes.

\section{Discussion}

Colibacillosis is caused by APEC and is considered one of the serious threats to the poultry industry and public health. APEC infections in birds cause many different kinds of clinical manifestations, ranging from respiratory tract infections to swollen head syndrome, which leads to death [12]. Although previous studies have reported the epidemic characteristics and pathogenic mechanism of APEC strains, detailed data on serotype, VAGs and molecular characteristics are often unavailable in many regions of China. Given that the zoonotic potential of APEC strains is still questionable, a considerable number of strains were isolated from chickens affected with colibacillosis, and several characteristics were compared between APEC and AFEC isolates in the present study.

APEC isolates often have diverse serotypes, and some overlapping serogroups are commonly detected in AFEC isolates. Although different $\mathrm{O}$ serogroups have been associated with colibacillosis, certain specific serogroups $(078,02$, and 01$)$ are more frequently reported than others $[11,17]$. Here, 078 was the most predominant serogroup, followed by 02 , which is similar to the results from previous studies. 01, however, was the sixth most frequently observed serotype among the 
APEC isolates in this investigation, and it was significantly higher than that in the AFEC isolates. 018 and 08 were also major serogroups, although the results have been controversial in different studies [12, 18].

APEC strains usually carry a large number of VAGs with different functions, and more VAGs in the same strain are often detected among E. coli from lesions [19-20]. Similar results were presented in our findings, and significantly more APEC isolates than AFEC isolates were collected from lesions. O-antigen is well proven to be the virulence factor of E. coli and can protect the bacteria from clearance by the neutrophils and macrophages of the host [21]. It is clear from the results of this study and previous evidence that serogroups 078 and 02 often possess more virulence and are considered virulent. However, different strains from the same serotype may vary in their virulence [18].

APEC strains are characterized by the possession of several VAGs, which enable these bacteria to survive an extraintestinal life and to cause colibacillosis [22-23]. In recent years, the mechanisms behind the pathogenesis and epidemic characteristics of VAGs of APEC strains have been extensively studied $[12,18]$. Some essential virulence genes, iroN (siderophore), ompT (outer membrane protease), iss (serum survival) and $h l y F$ (hemolysin), carried by plasmids are considered preferential molecular markers for APEC $[12,24]$. APEC isolates from poultry clinically diagnosed with colibacillosis were positive for at least one of these VAGs, and the frequencies vary greatly in different studies [11].

Sixteen VAGs were detected in the current study, and the frequencies of most VAGs were similar to those in these previous reports [11]. Among those VAGs, the frequencies of iro $\mathrm{N}$ and $o m p T$ were higher, while the frequencies of iucD, fyuA and vat were lower. Subedi et al. [25] reported an identical result: the virulence genes iro $N$ and ompT were harbored by each APEC isolate, but iss and hlyF were higher than those in the present study. We also found that all the AFEC isolates (carrying VAGs) were positive for both iroN and ompT.

It is speculated that the association of several different VAGs could increase the pathogenicity of bacteria [20]. The functions of virulence genes tested in the present study are well documented, and accumulation of these genes may be a potential risk factor for APEC infection. The epidemic characteristics of VAGs constitute unique VTs and evolution steps. Therefore, monitoring VTs with multiple VAGs in different hosts and understanding the evolution steps may be helpful for reducing economic losses in the poultry industry and the potential zoonotic risks of APEC strains[26].

Phylogenetic classification and MLST have several important advantages over PFGE, including shorter assay times, better standardization, and repeatability of data among laboratories. Epidemiological surveys in most previous studies have classified APEC strains as predominantly phylogroup B2, followed by phylogroup $D$, which are mainly responsible for extraintestinal infections and possess more VAGs [27]. The B2 group is closely related to pathogenicity and is frequently found among serogroups 078 and 02 isolates [28]. In the present study, the APEC isolates belonging to B2078/02-ST88 were the most associated with epidemics and carried more VAGs than the other strains. Our studies have suggested that there is a relationship among different E. coli phylogenetic groups, STs, serogroups and the virulence capabilities of the strains.

In conclusion, this study demonstrated the prevalence of common VAGs in APEC and AFEC strains recovered from colibacillosis tissue and fresh tissue. Our studies suggested that different VAGs have accumulated in APEC strains. However, the presence of these VAGs in AFEC isolates poses a potential risk of causing colibacillosis. Furthermore, the identification of predominant serotypes and molecular characteristics, which are closely related to pathogenicity, may be particularly useful in the diagnostic approach. Thus, regular screening and monitoring of APEC strains is essential for implementing intervention programs to reduce the risk of colibacillosis.

\section{Methods}

\section{Sample collection and bacterial isolation}


Animal-based active surveillance was conducted for 1568 tissue swab samples (liver, heart, lung and spleen), and 2799 fresh samples from infected chickens with typical lesions of an E. coli infection were collected from 26 different farms in Hebei Province from 2018 to 2020. All the samples were cultured on MacConkey agar overnight at $37^{\circ} \mathrm{C}$, and pinkcolored suspected bacterial colonies were further isolated on LB agar. For each sample, only one colony was isolated and used for subsequent examination.

The pure and presumptive positive strains were then confirmed as E. coli via biochemical analysis using the API20E system (bioMerieux, Marcy-l'Etoile, France) according to the manufacturer's recommendations. The isolates were stored at $-80^{\circ} \mathrm{C}$ in $25 \%$ glycerol.

\section{Preparation of DNA templates}

The DNA templates for PCR (serotype, VAGs, phylogroup, MLST) were directly extracted from bacterial colonies using the boiled lysate method. Briefly, a single colony from an overnight culture at $37^{\circ} \mathrm{C}$ on LB agar was suspended in $30 \mu \mathrm{L}$ sterile molecular grade water and boiled at $100^{\circ} \mathrm{C}$ for $10 \mathrm{~min}$. The sample was immediately cooled on ice for $5 \mathrm{~min}$ and centrifuged at $13,000 \mathrm{~g}$ at $4^{\circ} \mathrm{C}$ for $10 \mathrm{~min}$. The supernatant, containing DNA, was transferred to a fresh tube for use [29].

\section{Serological characterization}

Serotyping characterization was carried out using a multiplex PCR method for analyzing 162 different 0 antigens within 20 groups [30]. Multiplex PCR was performed as follows: each $30 \mu \mathrm{L}$ reaction mixture contained $2 \mu \mathrm{L}$ DNA template, 0.5 $\mu \mathrm{L}$ of each primer, $10 \mu \mathrm{L}$ Taq MasterMix (Takara, Japan) and deionized water to a final volume of $30 \mu \mathrm{L}$.

\section{Detection of virulence-associated genes}

All isolates also were screened for 5 virulence markers: papA and papC, sfa/foc, afa/dra, iutA, and kpsM II, for classification as ExPEC or non-ExPEC [31].

All strains identified as ExPEC were further tested by PCR for the presence of 16 virulence-associated genes [8, 11, 25, 32-34], including adhesins (fimC, papC, tsh, mat), invasins (ibeA), toxins (ast $A$, vat, hlyF), iron acquisition systems (iroN, fyuA, iucD, irp2), and protectins/serum resistance (iss, neuC, ompT, cva/cvi). PCRs were performed according to published protocols, and the primer sequences are listed in Table S3.

\section{Phylogenetic Classification}

The E. coli isolates were assigned to four phylogenetic groups (A, B1, B2, or D) based on three genes, ChuA and $Y j a A$ and an anonymous DNA fragment, TSPE4.C2, and a rapid and simple method as previously described [34], and the primers are shown in Table S4. The chuA and TspE4.C2-negative and TspE4.C2-positive E. coli strains were classified as groups $A$ and B2, respectively, and chuA-negative and TspE4.C2-positive, chuA-positive and yjaA-negative E. coli strains were grouped into B1 and D (Fig S1), respectively.

\section{Multilocus sequence typing (MLST)}

MLST analysis of 7 housekeeping genes was performed for each isolate according to the protocols described on the EcMLST website (http://www.shigatox.net/ecmlst). The PCR amplification conditions of the 7 housekeeping genes were as follows: $95^{\circ} \mathrm{C}$ for 5 min; 30 cycles of $94^{\circ} \mathrm{C}$ for $30 \mathrm{~s}, 55^{\circ} \mathrm{C}$ for $90 \mathrm{~s}$, and $72{ }^{\circ} \mathrm{C}$ for $1 \mathrm{~min}$; and $72{ }^{\circ} \mathrm{C}$ for 5 min with ExTaq DNA polymerase (Takara, Japan). The PCR products were bidirectionally sequenced, and the sequences of the 7 housekeeping genes were edited by using SeqMan 7.0. Each unique allele was assigned a different number, and the allelic profile (string of seven allelic loci) was used to define each isolate's sequence type (ST) [36]. Clustering analysis was used to infer relationships among the isolates using the fingerprint analysis software BioNumerics (version 7.1).

\section{Statistical Analysis}


The data for the APEC and AFEC isolates were analyzed using a chi-square test to find any significant differences. These differences were considered statistically significant when $\mathrm{P}<0.05$.

\section{Declarations}

\section{Ethics approval and consent to participate}

Ethical approval was granted for this study. Our study was conducted according to the Ethics Committee of Animal Experiments at the Institute of Husbandry and Pharmaceutical Sciences of Chinese Academy of Agricultural Sciences in Lanzhou, China. We gained consent from the owners of the animals for use in the study.

\section{Consent for publication}

All the authors agreed to the publication of the paper.

\section{Availability of data and material}

The data supporting the findings of this study are contained within the manuscript.

\section{Competing interests}

The authors declare that they have no competing interests.

\section{Funding}

The design of the study, including collection, analysis, and interpretation of the data, was supported by grants from the National Natural Science Foundation of China (No: 31872520) and Drug Development and Clinical Drug Use Posts of National Beef Yak Industry Technical System (No: CARS-37). The writing and submission of the manuscript was supported by the Natural Science Foundation of Hebei Province (No: C2019402114).

\section{Authors' contributions}

Z.Z. and J.Y.Z. designed the study; Z.Z., M.Z.C., Q.Q.Z., Y.X.S., X.Z.Z., C.Y.W. and G.B.C. generated and provided the dataset; Z.Z., M.Z.C., W.W.W., Y.Y.Z. and G.B.C. performed the experiments, analyzed the data, and wrote the manuscript. All authors have read and approved the final manuscript.

\section{Authors' information}

College of Life Science and Food Engineering, Hebei University of Engineering, Hanshan District, Handan, China

Key Laboratory of New Animal Drug Project of Gansu Province, Key Laboratory of Veterinary Pharmaceutical Development of Ministry of Agriculture, Lanzhou Institute of Husbandry and Pharmaceutical Sciences of CAAS, Jiangouyan, Qilihe District, Lanzhou, PR China

\section{References}

1. Ronco T, Stegger M, Olsen RH, Sekse C, Nordstoga AB, Pohjanvirta T, et al. Spread of avian pathogenic Escherichia coli ST117 078:H4 in Nordic broiler production. BMC Genomics. 2017;18(1):13.

2. Helmy YA, Deblais L, Kassem II, Kathayat D, Rajashekara G. Novel small molecule modulators of quorum sensing in avian pathogenic Escherichia coli (APEC). Virulence. 2018; 9(1):1640-1657. 
3. Alber A, Morris KM, Bryson KJ, Sutton KM, Monson MS, Chintoan-Uta C, et al. Avian Pathogenic Escherichia coli (APEC) Strain-Dependent Immunomodulation of Respiratory Granulocytes and Mononuclear Phagocytes in CSF1RReporter Transgenic Chickens. Front Immunol. 2020; 10:3055.

4. Schouler C, Schaeffer B, Brée A, Mora A, Dahbi G, Biet F, et al. Diagnostic strategy for identifying avian pathogenic Escherichia coli based on four patterns of virulence genes. J Clin Microbiol. 2012;50(5):1673-8.

5. Zhao S, Maurer JJ, Hubert S, De Villena JF, McDermott PF, Meng J, et al. Antimicrobial susceptibility and molecular characterization of avian pathogenic Escherichia coli isolates. Vet Microbiol. 2005;107(3-4):215-24.

6. Dziva F, Stevens MP. Colibacillosis in poultry: unravelling the molecular basis of virulence of avian pathogenic Escherichia coli in their natural hosts. Avian Pathol. 2008;37(4):355-66.

7. Olesen B. Characterization of four Escherichia coli clonal groups. APMIS. 2017;125 Suppl 139:1-28.

8. Maciel JF, Matter LB, Trindade MM, Camillo G, Lovato M, de Ávila Botton S, et al. Virulence factors and antimicrobial susceptibility profile of extraintestinal Escherichia coli isolated from an avian colisepticemia outbreak. Microb Pathog. 2017; 103: 119-122.

9. Díaz-Sánchez S, Sánchez S, Ewers C, Höfle U. Occurrence of avian pathogenic Escherichia coli and antimicrobialresistant E. coli in red-legged partridges (Alectoris rufa): sanitary concerns of farming. Avian Pathol. 2012,41(4):337-44.

10. Maluta RP, Logue CM, Casas MR, Meng T, Guastalli EA, Rojas TC, et al. Overlapped sequence types (STs) and serogroups of avian pathogenic (APEC) and human extra-intestinal pathogenic (ExPEC) Escherichia coli isolated in Brazil. PLoS One. 2014; 9(8):e105016.

11. Jeong YW, Kim TE, Kim JH, Kwon HJ. Pathotyping avian pathogenic Escherichia coli strains in Korea. J Vet Sci. 2012; 13(2):145-52.

12. Kim YB, Yoon MY, Ha JS, Seo KW, Noh EB, Son SH, et al. Molecular characterization of avian pathogenic Escherichia coli from broiler chickens with colibacillosis. Poult Sci. 2020; 99(2):1088-1095.

13. Koga VL, Rodrigues GR, Scandorieiro S, Vespero EC, Oba A, de Brito BG, et al. Evaluation of the antibiotic resistance and virulence of Escherichia coli strains isolated from chicken carcasses in 2007 and 2013 from Parana, Brazil. Foodborne Pathog Dis. 2015; 12: 479-485.

14. Cunha MPV, Saidenberg AB, Moreno AM, Ferreira AJP, Vieira MAM, Gomes TAT, et al. Pandemic extra-intestinal pathogenic Escherichia coli (ExPEC) clonal group 06-B2-ST73 as a cause of avian colibacillosis in Brazil. PLoS One. 2017;12(6):e0178970.

15. Manges AR. Escherichia coli and urinary tract infections: the role of poultry-meat. Clin Microbiol Infect. 2016;22(2):122-129.

16. Mellata M. Human and avian extraintestinal pathogenic Escherichia coli: infections, zoonotic risks, and antibiotic resistance trends. Foodborne Pathog Dis. 2013; 10(11):916-32.

17. Azam M, Mohsin M, Johnson TJ, Smith EA, Johnson A, Umair M, et al. Genomic landscape of multi-drug resistant avian pathogenic Escherichia coli recovered from broilers. Vet Microbiol. 2020; 247: 108766.

18. Paixão AC, Ferreira AC, Fontes M, Themudo P, Albuquerque T, Soares MC, et al. Detection of virulence-associated genes in pathogenic and commensal avian Escherichia coli isolates. Poult Sci. 2016;95(7):1646-1652.

19. Xu X, Sun Q, Zhao L. Virulence Factors and Antibiotic Resistance of Avian Pathogenic Escherichia Coli in Eastern China. J Vet Res. 2019;63(3):317-320.

20. Circella E, Pennelli D, Tagliabue S, Camarda A. Virulence-associated genes in avian pathogenic Escherichia coli from laying hens in Apulia, Southern Italy. Br Poult Sci. 2012; 53(4):465-70.

21. Crémet L, Broquet A, Jacqueline C, Chaillou C, Asehnoune K, Corvec S, et al. Innate immune evasion of Escherichia coli clinical strains from orthopedic implant infections. Eur J Clin Microbiol Infect Dis. 2016;35(6):993-9. 
22. De Carli S, Ikuta N, Lehmann FK, da Silveira VP, de Melo Predebon G, Fonseca AS, et al. Virulence gene content in Escherichia coli isolates from poultry flocks with clinical signs of colibacillosis in Brazil. Poult Sci. 2015;94:263540.

23. Johnson TJ, Wannemuehler Y, Doetkott C, Johnson SJ, Rosenberger SC, Nolan LK. Identification of minimal predictors of avian pathogenic Escherichia coli virulence for use as a rapid diagnostic tool. J Clin Microbiol. 2008;46(12):3987-96.

24. Jørgensen SL, Stegger M, Kudirkiene E, Lilje B, Poulsen LL, Ronco T, et al. Diversity and Population Overlap between Avian and Human Escherichia coli Belonging to Sequence Type 95. mSphere. 2019;4(1): e00333-18.

25. Subedi M, Luitel H, Devkota B, Bhattarai RK, Phuyal S, Panthi P, et al. Antibiotic resistance pattern and virulence genes content in avian pathogenic Escherichia coli (APEC) from broiler chickens in Chitwan, Nepal. BMC Vet Res. 2018; 14(1):113.

26. Johnson TJ, Wannemuehler Y, Johnson SJ, Stell AL, Doetkott C, Johnson JR, et al. Comparison of extraintestinal pathogenic Escherichia coli strains from human and avian sources reveals a mixed subset representing potential zoonotic pathogens. Appl Environ Microbiol. 2008; 74, 7043-7050.

27. Johnson JR, Stell AL. Extended virulence genotypes of Escherichia coli strains from patients with urosepsis in relation to phylogeny and host compromise. J Infect Dis. 2000;181:261-272.

28. Ciesielczuk H, Jenkins C, Chattaway M, Doumith M, Hope R, Woodford N, et al. Trends in ExPEC serogroups in the UK and their significance. Eur J Clin Microbiol Infect Dis. 2016; 35:1661-1666.

29. Ahmed AM, Shimamoto T, Shimamoto T. Molecular characterization of multidrug-resistant avian pathogenic Escherichia coli isolated from septicemic broilers. Int J. Med Microbiol. 2013; 303:475-483.

30. Iguchi A, lyoda S, Seto K, Morita-Ishihara T, Scheutz F, Ohnishi M, et al. Escherichia coli O-Genotyping PCR: a Comprehensive and Practical Platform for Molecular O Serogrouping. J Clin Microbiol. 2015; 53(8):2427-32.

31. Johnson J, Kuskowski M, Owens K, Gajewski A, Winokur P. Phylogenetic origin and virulence genotype in relation to resistance to fluoroquinolones and/or extended-spectrum cephalosporins and cephamycins among Escherichia coli isolates from animals and humans. J Infect Dis.2003 Sep 1;188(5):759 - 68.

32. Ewers C, Janssen T, Kiessling S, Philipp HC, Wieler LH. Rapid detection of virulence-associated genes in avian pathogenic Escherichia coli by multiplex polymerase chain reaction. Avian Dis. 2005;49(2):269-73.

33. Ewers C, Li G, Wilking H, Kiessling S, Alt K, Antáo EM, et al. Avian pathogenic, uropathogenic, and newborn meningitis-causing Escherichia coli: how closely related are they? Int J Med Microbiol. 2007;297(3):163-76.

34. Johnson TJ, Siek KE, Johnson SJ, Nolan LK. DNA sequence of a ColV plasmid and prevalence of selected plasmidencoded virulence genes among avian Escherichia coli strains. J Bacteriol. 2006;188(2):745-58.

35. Clermont O, Bonacorsi S, Bingen E. Rapid and simple determination of the Escherichia coli phylogenetic group. Appl Environ Microbiol. 2000;66(10):4555-8.

36. Schaumburg F, Alabi AS, Kaba H, Lell B, Becker K, Grobusch MP, et al. Molecular characterization of Shigella spp. from patients in Gabon 2011-2013. Trans R Soc Trop Med Hyg. 2015;109(4):275-9.

\section{Figures}


A
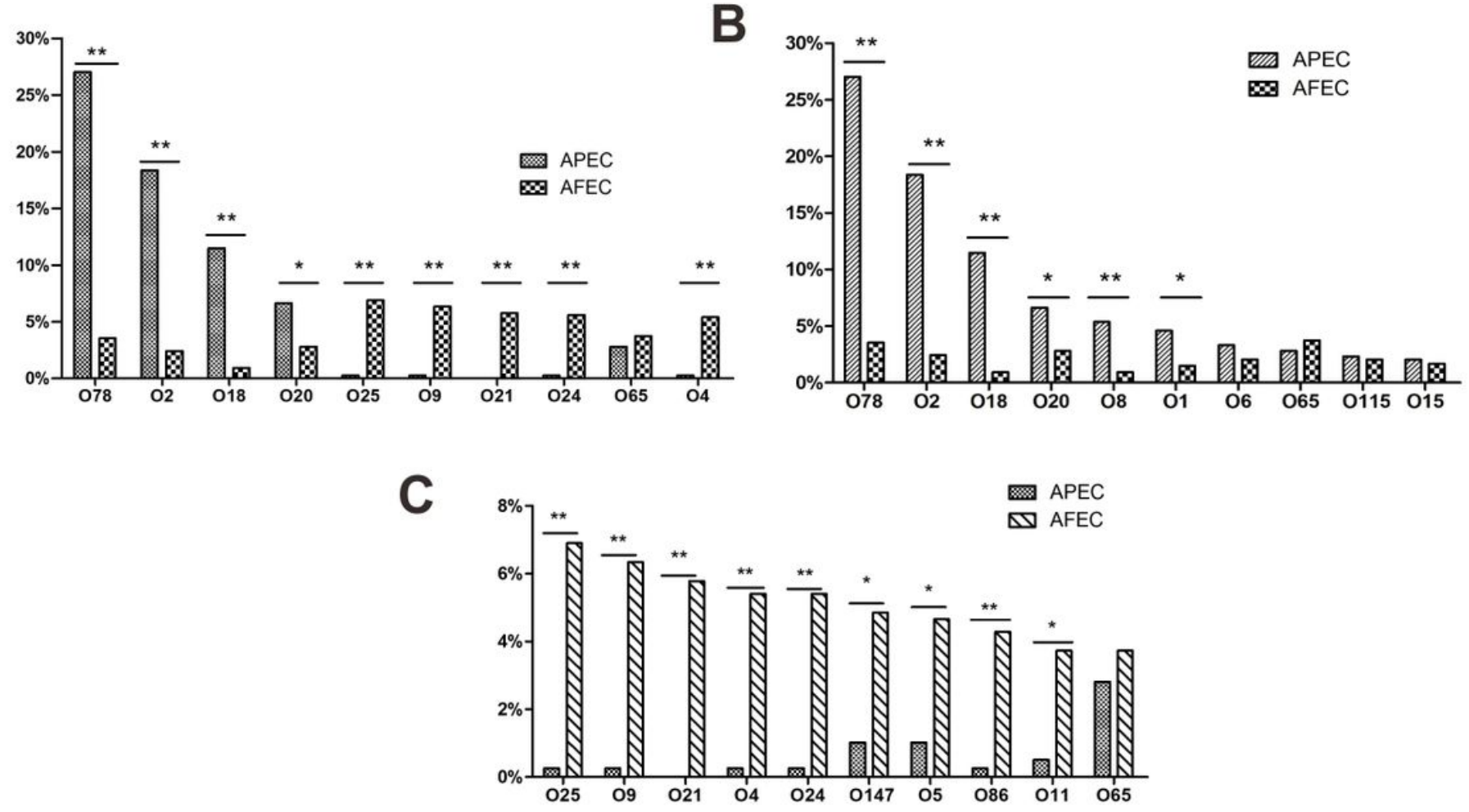

Figure 1

Distribution of the most predominant serotypes in E. coli. A: The 10 most predominant serotypes in total strains; B: The 10 most predominant serotypes in the APEC strains; C: The 10 most predominant serotypes in the AFEC strains. 


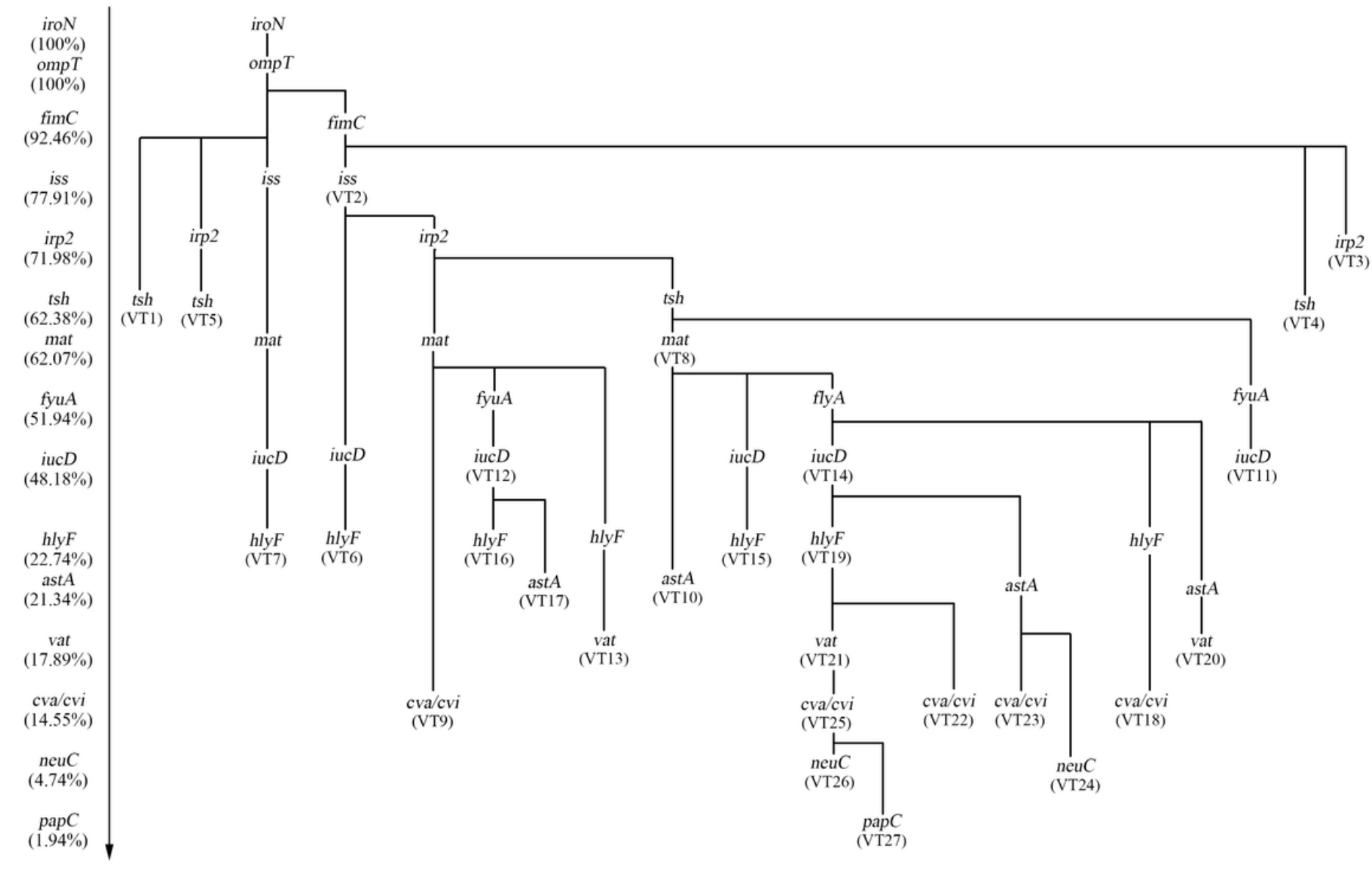

Figure 2

Accumulation of virulence genes and evolution of VTs in E. coli. According to the virulence gene frequencies among the VTs, the hypothetical steps of virulence gene acquisition were illustrated. 


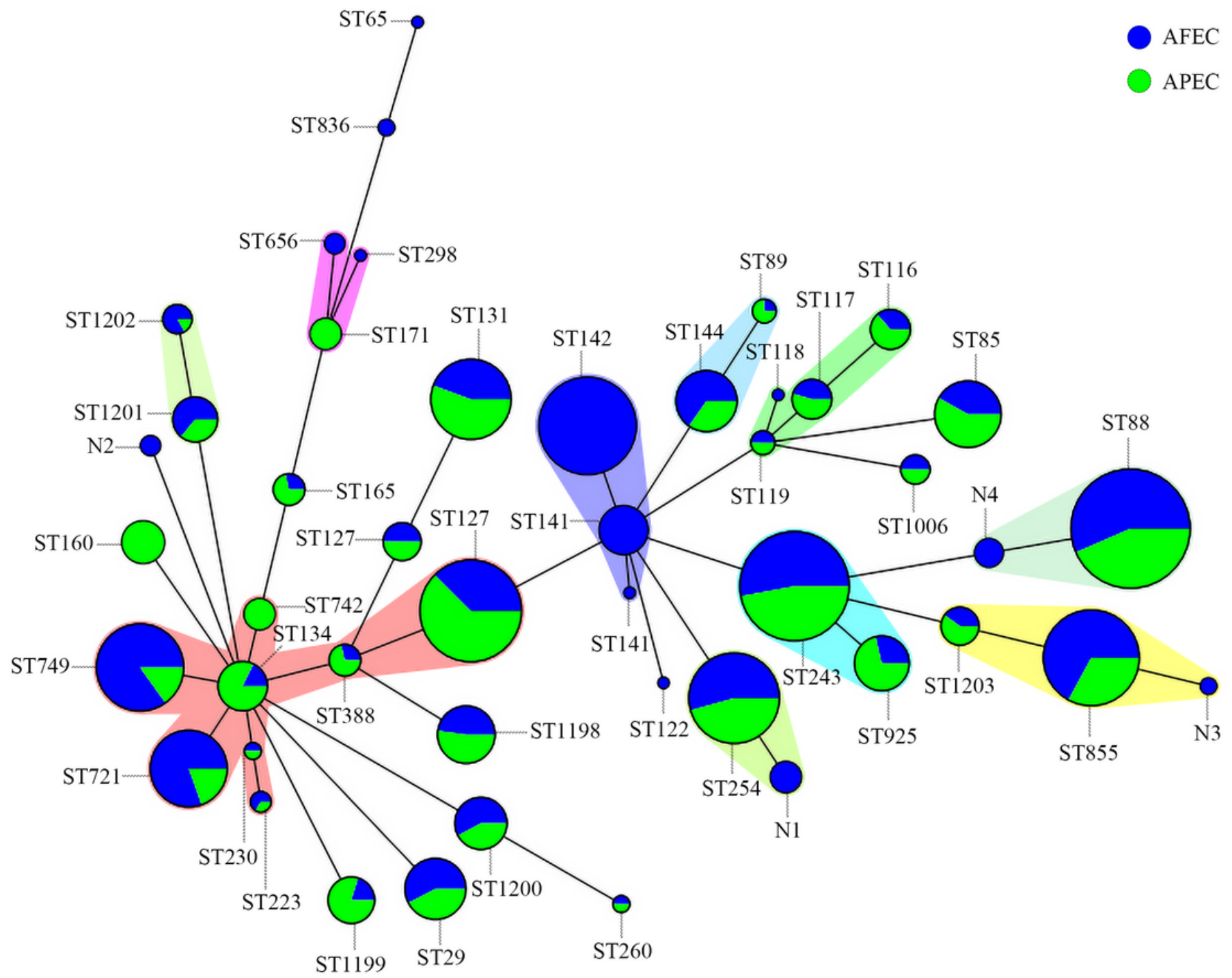

Figure 3

Minimum spanning tree of the $938 \mathrm{E}$. coli isolates from chickens based on multilocus sequence typing (MLST). The minimum spanning tree was constructed using the 7 identified STs obtained from the 938 isolates using BioNumerics Software. Each circle corresponds to a single ST. The shadow zones in different colors correspond to different E.coli (APEC or AFEC).

A

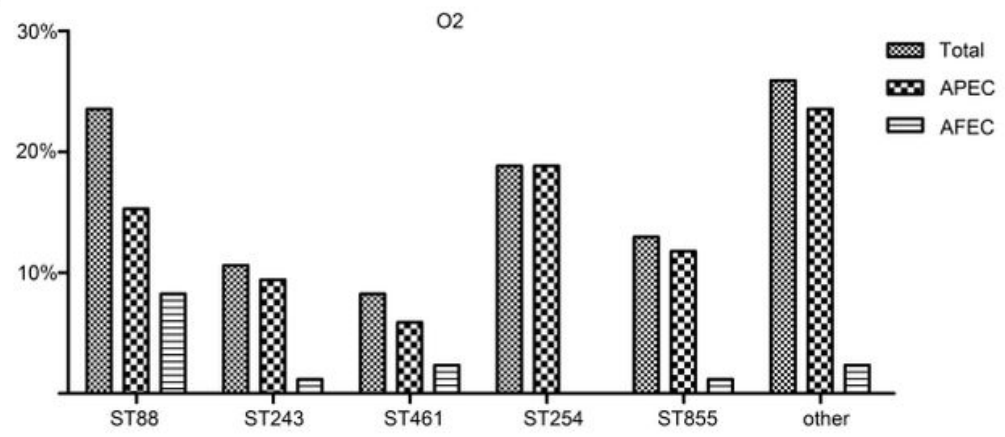

B

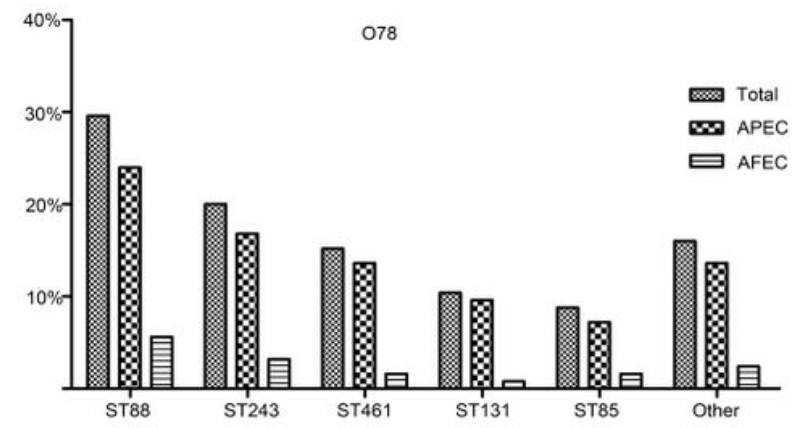


The major STs in $078(A)$ and $02(B)$ serotype strains.

\section{Supplementary Files}

This is a list of supplementary files associated with this preprint. Click to download.

- Figs1.tiff

- Tables1.docx

- Tables2.docx

- Tables3.docx

- Tables4..docx 